\title{
Changes in FGF-23, Neutrophil/Platelet Activation Markers, and Angiogenin in Advanced Chronic Kidney Disease and Their Effect on Arterial Stiffness
}

\author{
Hye-Min Choi Young-Eun Kwon Sol Kim Dong-Jin Oh \\ Division of Nephrology, Department of Internal Medicine, Myongji Hospital, Hanyang \\ University College of Medicine, Goyang, South Korea
}

\section{Keywords}

Arterial stiffness · Chronic kidney disease - Neutrophil activation markers · Angiogenin ·

Fibroblast growth factor $23 \cdot$ Mean platelet volume-to-platelet count ratio

\begin{abstract}
Aims: The aims of this study were to measure changes in fibroblast growth factor 23 (FGF-23), neutrophil (elastase, lactoferrin)/platelet activation marker (mean platelet volume-to-platelet count ratio $[M P R])$, and angiogenin according to the stage of chronic kidney disease (CKD), and to evaluate the association of FGF-23, elastase, lactoferrin, MPR, and angiogenin with arterial stiffness using brachial-ankle pulse wave velocity (ba-PWV) in CKD patients. Methods: According to the estimated glomerular filtration rate (eGFR) calculated using the Chronic Kidney Disease Epidemiology Collaboration (CKD-EPI) equation, the patients were allocated to five groups: (1) normal controls (eGFR $\geq 90 \mathrm{~mL} / \mathrm{min} / 1.73 \mathrm{~m}^{2}$ without pathologic, urine [proteinuria], blood [electrolyte], and imaging abnormalities; $n=22)$; (2) CKD stage 2 (eGFR 60-89 mL/min/1.73 m²; $n=17$ ); (3) CKD stage 3 (eGFR 30-59 mL/min/1.73 m²; $n=22$ ); (4) CKD stage 4 (eGFR 15-30 mL/ $\left.\min / 1.73 \mathrm{~m}^{2} ; n=17\right)$; and (5) CKD stage 5-hemodialysis (HD) $(n=30)$. All the patients were free of clinically apparent cardiovascular disease. Serum FGF-23, elastase, lactoferrin, and angiogenin concentrations and the MPR were measured to study the association of the above parameters with the clinical (age, sex, presence of diabetes mellitus, and blood pressure), biochemical (calcium, phosphorus, uric acid, intact parathyroid hormone [PTH], low-density lipoprotein cholesterol, and high-sensitivity C-reactive protein), and ba-PWV values of the CKD patients. Results: (1) The mean ba-PWV values were 1,497.2 $\pm 206.4 \mathrm{~cm} / \mathrm{s}$ in the controls, $1,649.0 \pm 247.9 \mathrm{~cm} / \mathrm{s}$ in the CKD stage 2 group ( $p<0.05$ vs. controls), 1,655.8 $\pm 260.3 \mathrm{~cm} / \mathrm{s}$ in the CKD stage 3 group ( $p<0.05$ vs. controls), 1,823.0 $\pm 402.4 \mathrm{~cm} / \mathrm{s}$ in the CKD stage 4 group
\end{abstract}




\section{Kidney \\ Blood Pressure \\ Research}

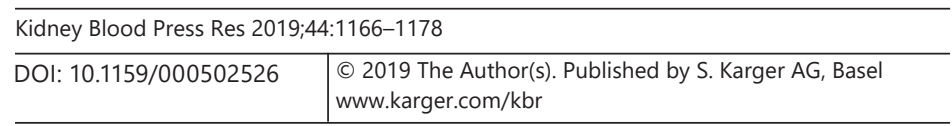

( $p<0.05$ vs. controls and CKD stages 2 and 3), and 1,905.2 $\pm 374.1 \mathrm{~cm} / \mathrm{s}$ in the CKD stage $5-\mathrm{HD}$ group ( $p<0.05$ vs. controls and CKD stage 2). (2) The mean $\log _{10}$ (FGF-23) concentration values were $0.77 \pm 0.27,0.97 \pm 0.48,1.10 \pm 0.35$ ( $p<0.05$ vs. controls and CKD stage 2$), 1.35 \pm 0.48$ ( $p<0.05$ vs. controls and CKD stages 2 and 3), and $2.12 \pm 0.82$ ( $p<0.05$ vs. controls and CKD stages $2-4)$; the mean angiogenin levels were $230.6 \pm 70.5 \mathrm{pg} / \mathrm{mL}, 283.0 \pm 53.5 \mathrm{pg} / \mathrm{mL}(p<0.05$ vs. controls), $347.3 \pm 76.9 \mathrm{pg} / \mathrm{mL}$ ( $p<0.05$ vs. controls and CKD stage 2$), 445.9 \pm 90.6 \mathrm{pg} / \mathrm{mL}$ ( $p<0.05$ vs. controls and CKD stages 2 and 3 ), and $370.9 \pm 142.4 \mathrm{pg} / \mathrm{mL}(p<0.05$ vs. controls and CKD stages 2 and 3). (3) In the stage 3-4 CKD/HD patients, the mean elastase-to-neutrophil and lactoferrin-to-neutrophil ratios were significantly lower than in the controls and the stage 2 CKD patients. (4) Our multivariate linear regression analyses showed that age, pulse pressure, mean arterial pressure, PTH, and FGF-23 were independently associated with ba-PWV values. Conclusions: Circulating FGF-23 and angiogenin concentrations gradually increased as CKD advanced, whereas neutrophil activation markers were significantly lower in the stage 3-4 CKD/ HD patients than in the controls and stage 2 CKD patients. FGF-23 was weakly associated with ba-PWV values in patients with CKD/HD and no previous cardiovascular disease.

(C) 2019 The Author(s)

Published by S. Karger AG, Basel

\section{Introduction}

The growing prevalence of chronic kidney disease (CKD) is associated with the aging of populations. These patients have high morbidity and mortality rates that are mainly due to cardiovascular disease [1, 2]. Arterial stiffness (AS) has been identified as an independent predictor of all-cause and cardiovascular mortality in patients with CKD and end-stage renal disease [3].

Fibroblast growth factor 23 (FGF-23) is a circulating peptide hormone secreted by bone cells and has recently emerged as one of the most powerful predictors of adverse cardiovascular outcomes and CKD progression. Recently, serum FGF-23 levels have been found to increase as the CKD stages advance in patients with autosomal dominant polycystic kidney disease (ADPKD) [4]. Increased serum FGF-23 levels in ADPKD patients have also been associated with increased AS and atherosclerosis development, regardless of soluble Klotho [4]. Moreover, another report found that FGF-23 concentration was positively associated with AS in patients with type 1 diabetes mellitus and no previous cardiovascular disease [5].

The number of circulating neutrophils, neutrophil activation markers such as elastase and lactoferrin, and the oxidative status are increased in CKD [6]. The activation of neutrophils, with the enhanced release of reactive oxygen species (ROS), contributes to the development of oxidative stress; the release of proteases contributes to endothelial dysfunction and to an inflammatory response that is further enhanced by the release of proinflammatory cytokines. All these mechanisms contribute to a worsening of the vascular injury and to the development of AS [7-9].

Angiogenin, a 14.4-kDa single-chain basic protein, is a potent inducer of neovascularization; Burgmann et al. [10] found significantly increased levels of angiogenin in the serum of patients with stage IV peripheral arterial occlusive disease (PAOD) compared to control groups, patients with stage IIb PAOD, and patients with deep vein thrombosis. The increased production of angiogenin may be indicative of compensatory angiogenesis in these patients. Revascularization and the development of collaterals are important mechanisms of compensation in patients with PAOD.

The mean platelet volume (MPV), a determinant of platelet activation, was recognized as an independent risk factor for hypertension, myocardial infarction, and stroke [11]. Orscelik 


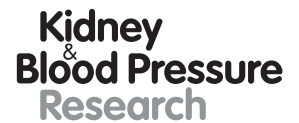

Kidney
Blood Pressure
Research

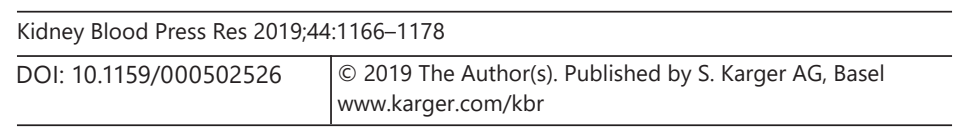

Choi et al.: FGF-23 in Arterial Stiffness

et al. [12] demonstrated that MPV was associated with increased AS in patients with earlystage ADPKD or late-stage ADPKD.

The reports mentioned above have suggested that FGF-23, neutrophil/platelet activation status, and angiogenin may be linked with AS in patients with CKD. However, to date, the levels of these mediators at the different stages of CKD have not been determined. As such, the possible association of FGF-23, neutrophil/platelet activation, and angiogenin with AS has not yet been fully evaluated. Therefore, the aim of the present study was to observe the changes in FGF-23, neutrophil/platelet activation marker, and angiogenin in the different stages of CKD, as well as to evaluate the association of FGF-23, neutrophil/platelet activation marker, and angiogenin with AS using brachial-ankle pulse wave velocity (ba-PWV) in patients with CKD/hemodialysis (HD).

\section{Patients and Methods}

\section{Study Population}

Of 110 patients undergoing HD in our artificial kidney center, we excluded the following patients from our study: (a) patients within 3 months of starting HD; (b) hemodynamically unstable subjects (those with frequent hypotension during HD); (c) patients with a previous history of ischemic or hemorrhagic stroke or heart disease including atrial fibrillation (any ischemic and arrhythmic evidence on ECG, a left ventricular ejection fraction $<45 \%$, valvular heart disorder, or cardiomyopathy on 2-D echocardiography); (d) patients with a previous history of cardiac intervention (coronary bypass, stent, or cardiac pacemaker) or renal transplantation; (e) patients with ADPKD; (f) patients with active infection or high-sensitivity C-reactive protein (hs-CRP) $>0.5 \mathrm{mg} / \mathrm{dL}$; (g) patients with PAOD, such as gangrene, and amputees; (h) patients undergoing percutaneous transluminal angioplasty and having an ankle-brachial index <0.95; (i) patients with a recent arteriovenous access event, such as thrombosis and infection, or intervention before recruitment; (j) patients with a urea Kt/V $<1.2$; (k) smokers; and (l) patients aged $<18$ years.

After screening, 30 patients who had been on HD for $\geq 3$ months (31 [3-108] months), 22 age/sex/nonsmoker-matched healthy control patients (estimated glomerular filtration rate [eGFR] $>90 \mathrm{~mL} / \mathrm{min} / 1.73 \mathrm{~m}^{2}$ without pathologic, urine [proteinuria], blood [electrolyte], and imaging abnormalities) who did not have any chronic disorder or history of medication use, 17 patients with stage 2 CKD (eGFR $60-89 \mathrm{~mL} / \mathrm{min} / 1.73 \mathrm{~m}^{2}$ ), 22 patients with stage $3 \mathrm{CKD}$ (eGFR $30-59 \mathrm{~mL} / \mathrm{min} / 1.73 \mathrm{~m}^{2}$ ), and 17 patients with stage 4 CKD (eGFR $15-29 \mathrm{~mL} / \mathrm{min} / 1.73$ $\mathrm{m}^{2}$ ) who were free of clinically apparent cardiovascular disease were recruited between January and December 2018. None of the control-to-stage 4 patients met any of the above exclusion criteria.

The eGFR was calculated using the Chronic Kidney Disease Epidemiology Collaboration (CKD-EPI) equation based on serum creatinine, age, sex, and race. The mean HD efficiency, as expressed by urea $\mathrm{Kt} / \mathrm{V}$, in our study population was $1.40 \pm 0.14$. The serum levels of elastase, lactoferrin, angiogenin, and FGF-23, as well as calcium (Ca), phosphorus (P), uric acid, intact parathyroid hormone (PTH), low-density lipoprotein (LDL) cholesterol, hs-CRP, blood pressure, neutrophils, platelets, and MPV were measured. Additionally, ba-PWV was evaluated.

\section{Biochemical Measures}

Blood samples were obtained prior to a mid-week session of HD or a routine outpatient clinic visit (for the control-to-stage 2-4 CKD patients). The blood samples were analyzed for $\mathrm{P}$ and Ca concentrations using a Hitachi 747 automated analyzer, with the ranges of 1.9-3.4 
and $8.2-10.4 \mathrm{mg} / \mathrm{dL}$, respectively, considered as normal. Intact PTH was measured using a commercial immunoradiometric assay (Diagnostic Products Corporation, Los Angeles, CA, USA), with a normal range of $12-72 \mathrm{pg} / \mathrm{mL}$. hs-CRP was measured using a COBAS INTEGRA analyzer (Roche Diagnostic Systems, Somerville, NJ, USA). Serum total cholesterol, LDL cholesterol, high-density lipoprotein cholesterol, and triglyceride levels were measured by enzymatic methods using a Hitachi 736-740 autoanalyzer. EDTA-containing tubes were used for complete blood counts, and the MPV was determined using a Sysmex XE-2100 system (Sysmex Corporation, Kobe, Japan) within 1-2 h after venous puncture and adjusted with a platelet count (MPV-to-platelet count ratio [MPR]).

Blood pressure was measured 3 consecutive times by the same nurse on each occasion using a conventional sphygmomanometer, with the subject in a sitting position. The mean arterial pressure (MAP) was calculated using the following formula: (systolic blood pressure $[\mathrm{SBP}]+2 \times$ diastolic blood pressure [DBP])/3. Pulse pressure (PP) was calculated using the following formula: SBP - DBP.

\section{Determination of Circulating FGF-23, Elastase, Lactoferrin, and \\ Angiogenin Concentrations}

Serum FGF-23 was measured using an ELISA kit (Immutopics Inc., San Clemente, CA, USA). The intra- and interassay coefficients of variation were 2.4 and $4.7 \%$ for FGF-23. Serum elastase and lactoferrin concentrations were determined using enzyme immunoassays (human PMN elastase ELISA, Bender MedSystems, and Lactoferrin ELISA Kit, Calbiochem, respectively). The elastase and lactoferrin concentrations were adjusted with a neutrophil count. The serum angiogenin concentration was measured using ELISA Quantikine 1 tests (R\&D Systems, USA), according to the manufacturer's instructions. Test sensitivity was $6 \mathrm{pg} / \mathrm{mL}$.

\section{Brachial-Ankle Pulse Wave Velocity}

The development of AS was determined by ba-PWV for the entire patient cohort, including the control groups, after 15 min resting in the supine position, using an ankle-brachial indexmeasuring instrument (I.E.M. GmbH, Stolberg, Germany), as previously described [13]. The blood pressure measurements in both the arms and the legs were calculated automatically and at the same time. PWV was calculated by determining the delay between the localized blood pressure curves at two different points of the arterial system. The interval between the start and the end of the brachial-ankle waves obtained from the brachial and tibial arteries was determined as the transition time "TT". The transition distance between the brachium and the ankle was determined according to weight. The distance between the suprasternal notch and the brachium "LB" was calculated using the following formula: $0.2195 \times$ height of the patient (in $\mathrm{cm}$ ) - 2.0734. The distance between the suprasternal notch and the ankle "LA" was calculated using the following formula: $0.2195 \times$ height of the patient (in $\mathrm{cm})+12.328$. The ba-PWV value was determined using the following formula: (LA - LB)/TT.

\section{Statistical Analysis}

Variables with Gaussian or skewed distributions are expressed as the mean \pm standard deviation (SD) or mean \pm standard error of the mean (SEM), respectively. Between-group differences were assessed for significance using unpaired Student's $t$ tests and Mann-Whitney U tests, as appropriate. Pearson's (parametric) correlations were used to test for associations of $\log _{10}$ (FGF-23) (due to skewed distribution of serum FGF-23 concentrations), elastase/ lactoferrin-to-neutrophil ratio, MPR, and angiogenin with selected clinical, anthropometric, biochemical, and vascular measures. Comparisons between the patient and control groups were performed using one-way analyses of variance. Multivariate linear regression analyses 
were performed to test the associations of $\log _{10}$ (FGF-23), elastase/lactoferrin-to-neutrophil ratio, MPR, and angiogenin with outcome variables, including ba-PWV. The statistical analyses were performed using SPSS software version 14.0 (SPSS Inc., Chicago, IL, USA).

\section{Results}

The study included a total of 108 patients from control to stage 2-4 CKD/HD with a mean age of $63.7 \pm 9.3$ years; $54(50.0 \%)$ of them were men. The mean SBP was $128.9 \pm 15.1 \mathrm{~mm}$ $\mathrm{Hg}$, and the mean DBP was $74.4 \pm 12.7 \mathrm{~mm} \mathrm{Hg}$. Forty-four patients $(40.7 \%)$ had diabetes mellitus and 56 patients (52.0\%) had hypertension. Antihypertensive medications used by the patients included an angiotensin-converting enzyme inhibitor in 9 cases (17.4\%), an angiotensin II receptor blocker in 18 cases (31.4\%), a calcium channel blocker in 13 cases (23.3\%), an alpha-blocker in 3 cases (4.7\%), a beta-blocker in 9 cases $(17.4 \%)$, a diuretic in 3 cases $(4.7 \%)$, and other antihypertensive medications in 1 case $(1.1 \%)$.

Nineteen (40.4\%) and 14 (29.8\%) of the stage 4-to-stage 5-HD CKD patients (47 patients) were administered phosphorus binders and active vitamin D metabolites (cinacalcet and/or paricalcitol), respectively. The mean creatinine level was $2.0 \pm 1.1 \mathrm{mg} / \mathrm{dL}$, and the mean eGFR was $34.1 \pm 25.3 \mathrm{~mL} / \mathrm{min} / 1.73 \mathrm{~m}^{2}$. The $\mathrm{Ca}, \mathrm{P}, \mathrm{Ca} \times \mathrm{P}$ product, $\mathrm{PTH}$, uric acid, LDL cholesterol, and hs-CRP values were $9.1 \pm 0.5 \mathrm{mg} / \mathrm{dL}, 4.1 \pm 1.2 \mathrm{mg} / \mathrm{dL}, 37.0 \pm 15.6,138.7 \pm 149.4 \mathrm{pg} / \mathrm{mL}$, $5.9 \pm 5.6 \mathrm{mg} / \mathrm{dL}, 81.7 \pm 31.5 \mathrm{mg} / \mathrm{dL}$, and $0.2 \pm 0.1 \mathrm{mg} / \mathrm{dL}$, respectively. The mean serum elastase, elastase-to-neutrophil ratio, lactoferrin, lactoferrin-to-neutrophil ratio, angiogenin, $\log _{10}$ (FGF-23), PTH-to-FGF-23 ratio, and (PTH $\times$ FGF-23 product) $/ 1,000$ values were $643.2 \pm$ $426.1 \mathrm{ng} / \mathrm{mL}, 193.7 \pm 127.6,357.0 \pm 236.2 \mathrm{ng} / \mathrm{mL}, 109.1 \pm 72.1,327.9 \pm 121.1 \mathrm{pg} / \mathrm{mL}, 1.3 \pm$ $0.8 \mathrm{pg} / \mathrm{mL}, 5.4 \pm 5.7$, and $113.6 \pm 494.9 \mathrm{pg}^{2} / \mathrm{mL}^{2}$, respectively. The MPV, platelet count, and MPR were $10.8 \pm 9.0 \mathrm{fL}, 221.0 \pm 71.4 \times 1,000 / \mathrm{mm}^{3}$, and $0.055 \pm 0.064$. The mean ba-PWV was $1,656.9 \pm 306.2 \mathrm{~cm} / \mathrm{s}$ (Table 1 ).

Compared to the healthy controls, the patients at the various predialysis CKD stages had significantly higher values for $(\mathrm{PTH} \times \mathrm{FGF}-23$ product $) / 1,000, \mathrm{PP}, \mathrm{P}, \mathrm{Ca} \times \mathrm{P}$ product, PTH, and uric acid, and significantly lower values for PTH-to-FGF-23 ratio, Ca, LDL cholesterol, and platelet count. There was no significant difference between the groups regarding age, sex, presence of diabetes mellitus, SBP, DBP, MAP, hs-CRP, neutrophil count, and MPV (Table 2).

The mean ba-PWV values in the control to stage 5 -HD groups were $1,497.2 \pm 206.4 \mathrm{~cm} / \mathrm{s}$, $1,649.0 \pm 247.9 \mathrm{~cm} / \mathrm{s}(p<0.05$ vs. controls), $1,655.8 \pm 260.3 \mathrm{~cm} / \mathrm{s}(p<0.05$ vs. controls $)$, $1,823.0 \pm 402.4 \mathrm{~cm} / \mathrm{s}$ ( $p<0.05$ vs. controls and CKD stages 2 and 3$)$, and 1,905.2 $\pm 374.1 \mathrm{~cm} / \mathrm{s}$ ( $p<0.05$ vs. controls and CKD stages 2 and 3) (Table 2). The mean MPRs in the control to stage 5 -HD groups were $0.046 \pm 0.014,0.039 \pm 0.012,0.047 \pm 0.011,0.048 \pm 0.016$, and $0.059 \pm$ 0.020 ( $p<0.05$ vs. controls and CKD stages $2-4$ ) (Table 2).

The mean $\log _{10}$ (FGF-23) concentrations in the control to stage 5 -HD groups were $0.77 \pm$ $0.27,0.97 \pm 0.48,1.10 \pm 0.35$ ( $p<0.05$ vs. controls and CKD stage 2$), 1.35 \pm 0.48$ ( $p<0.05$ vs. controls and CKD stages 2 and 3 ), and $2.12 \pm 0.82$ ( $p<0.05$ vs. controls and CKD stages $2-4$ ) (Fig. 1).

The mean angiogenin levels in the control to stage 5-HD groups were $230.6 \pm 70.5 \mathrm{pg} /$ $\mathrm{mL}, 283.0 \pm 53.5 \mathrm{pg} / \mathrm{mL}(p<0.05$ vs. controls), $347.3 \pm 76.9 \mathrm{pg} / \mathrm{mL}$ ( $p<0.05 \mathrm{vs}$. controls and CKD stage 2), $445.9 \pm 90.6 \mathrm{pg} / \mathrm{mL}$ ( $p<0.05$ vs. controls and CKD stages 2 and 3 ), and $370.9 \pm$ $142.4 \mathrm{pg} / \mathrm{mL}(p<0.05$ vs. controls and CKD stages 2 and 3) (Fig. 2).

The mean elastase-to-neutrophil and lactoferrin-to-neutrophil ratios in the control to stage 5-HD groups were $274.9 \pm 164.3$ and $156.2 \pm 95.4,260.9 \pm 170.0$ and $149.2 \pm 110.1$, $161.7 \pm 69.4$ and $83.9 \pm 30.7$ (both $p<0.05$ vs. controls and CKD stage 2), $125.1 \pm 64.9$ and 
Kidney

Blood Pressure

Research

Table 1. Characteristics of the study population $(n=108)$

\begin{tabular}{l|l}
\hline Kidney Blood Press Res 2019;44:1166-1178 \\
\hline DOI: 10.1159/000502526 & $\begin{array}{l}\text { @ 2019 The Author(s). Published by S. Karger AG, Basel } \\
\text { www.karger.com/kbr }\end{array}$ \\
\hline
\end{tabular}

Choi et al.: FGF-23 in Arterial Stiffness

\begin{tabular}{|c|c|}
\hline Age, years & $63.7 \pm 9.3$ \\
\hline Males, \% & 50.0 \\
\hline $\mathrm{DM}, \%$ & 40.7 \\
\hline Creatinine, $\mathrm{mg} / \mathrm{dL}$ & $2.0 \pm 1.1$ \\
\hline eGFR by CKD-EPI equation, $\mathrm{mL} / \mathrm{min} / 1.73 \mathrm{~m}^{2}$ & $34.1 \pm 25.3$ \\
\hline Neutrophils, $\times 10^{9} / \mathrm{L}$ & $3.6 \pm 1.4$ \\
\hline Elastase, $\mathrm{ng} / \mathrm{mL}$ & $643.2 \pm 426.1$ \\
\hline Elastase-to-neutrophil ratio & $193.7 \pm 127.6$ \\
\hline Lactoferrin, ng/mL & $357.0 \pm 236.2$ \\
\hline Lactoferrin-to-neutrophil ratio & $109.1 \pm 72.1$ \\
\hline Angiogenin, pg/mL & $327.9 \pm 121.1$ \\
\hline $\log _{10}(\mathrm{FGF}-23), \mathrm{pg} / \mathrm{mL}$ & $1.3 \pm 0.8$ \\
\hline PTH-to-FGF-23 ratio & $5.4 \pm 5.7$ \\
\hline$(\mathrm{PTH} \times \mathrm{FGF}-23$ product $) / 1,000$ & $113.6 \pm 494.9$ \\
\hline $\mathrm{SBP}, \mathrm{mm} \mathrm{Hg}$ & $128.9 \pm 15.1$ \\
\hline DBP, mm Hg & $74.4 \pm 12.7$ \\
\hline MAP, mm Hg & $92.5 \pm 12.1$ \\
\hline $\mathrm{PP}, \mathrm{mm} \mathrm{Hg}$ & $54.5 \pm 14.0$ \\
\hline $\mathrm{Ca}, \mathrm{mg} / \mathrm{dL}$ & $9.1 \pm 0.5$ \\
\hline $\mathrm{P}, \mathrm{mg} / \mathrm{dL}$ & $4.1 \pm 1.2$ \\
\hline $\mathrm{Ca} \times \mathrm{P}$ product & $37.0 \pm 15.6$ \\
\hline PTH, pg/mL & $138.7 \pm 149.4$ \\
\hline Uric acid, mg/dL & $5.9 \pm 5.6$ \\
\hline LDL cholesterol, mg/dL & $81.7 \pm 31.5$ \\
\hline hs-CRP, mg/dL & $0.2 \pm 0.1$ \\
\hline $\mathrm{MPV}, \mathrm{fL}$ & $10.8 \pm 9.0$ \\
\hline Platelet count, $\times 1,000 / \mathrm{mm}^{3}$ & $221.0 \pm 81.0$ \\
\hline MPR & $0.055 \pm 0.064$ \\
\hline ba-PWV, cm/s & $1,656.9 \pm 306.2$ \\
\hline
\end{tabular}

Data are expressed as the mean \pm SD or $\%$. DM, diabetes mellitus; eGFR, estimated glomerular filtration rate; CKD-EPI, Chronic Kidney Disease Epidemiology Collaboration; FGF-23, fibroblast growth factor 23; PTH, intact parathyroid hormone; SBP, systolic blood pressure; $\mathrm{DBP}$, diastolic blood pressure; MAP, mean arterial pressure; PP, pulse pressure; Ca, calcium; P, phosphorus; LDL cholesterol, low-density lipoprotein cholesterol; hs-CRP, high-sensitivity C-reactive protein; MPV, mean platelet volume; MPR, mean platelet volume-to-platelet count ratio; ba-PWV, brachial-ankle pulse wave velocity.

$66.9 \pm 23.0$ (both $p<0.05$ vs. controls and CKD stage 2), and $138.4 \pm 75.9$ and $85.9 \pm 43.2$ (both $p<0.05$ vs. controls and CKD stage 2), respectively (Fig. 3, 4).

The ba-PWV values showed a positive correlation with age $(r=0.487, p=0.001)$, CKD stage $(r=0.439, p=0.001), \operatorname{SBP}(r=0.379, p=0.001), \operatorname{MAP}(r=0.256, p=0.012), \operatorname{PP}(r=0.294$, $p=0.005)$, PTH $(r=0.381, p=0.001)$, FGF-23 $(r=0.243, p=0.016), \mathrm{PTH} \times \mathrm{FGF}-23$ product $(r=0.200, p=0.040), \mathrm{P}(r=0.250, p=0.014)$, and Ca $\times \mathrm{P}$ product $(r=0.222, p=0.025)$. However, there was no correlation of serum elastase/lactoferrin-to-neutrophil ratio, MPR, and angiogenin concentration with ba-PWV values (Table 3).

The multivariate linear regression analysis demonstrated that age $(\beta=16.405, p=0.001)$, PP ( $\beta=7.264, p=0.001)$, MAP $(\beta=5.535, p=0.035)$, PTH ( $\beta=0.859, p=0.029)$, and FGF-23 $(\beta=0.213, p=0.049)$ were independently associated with ba-PWV values (Table 4). 


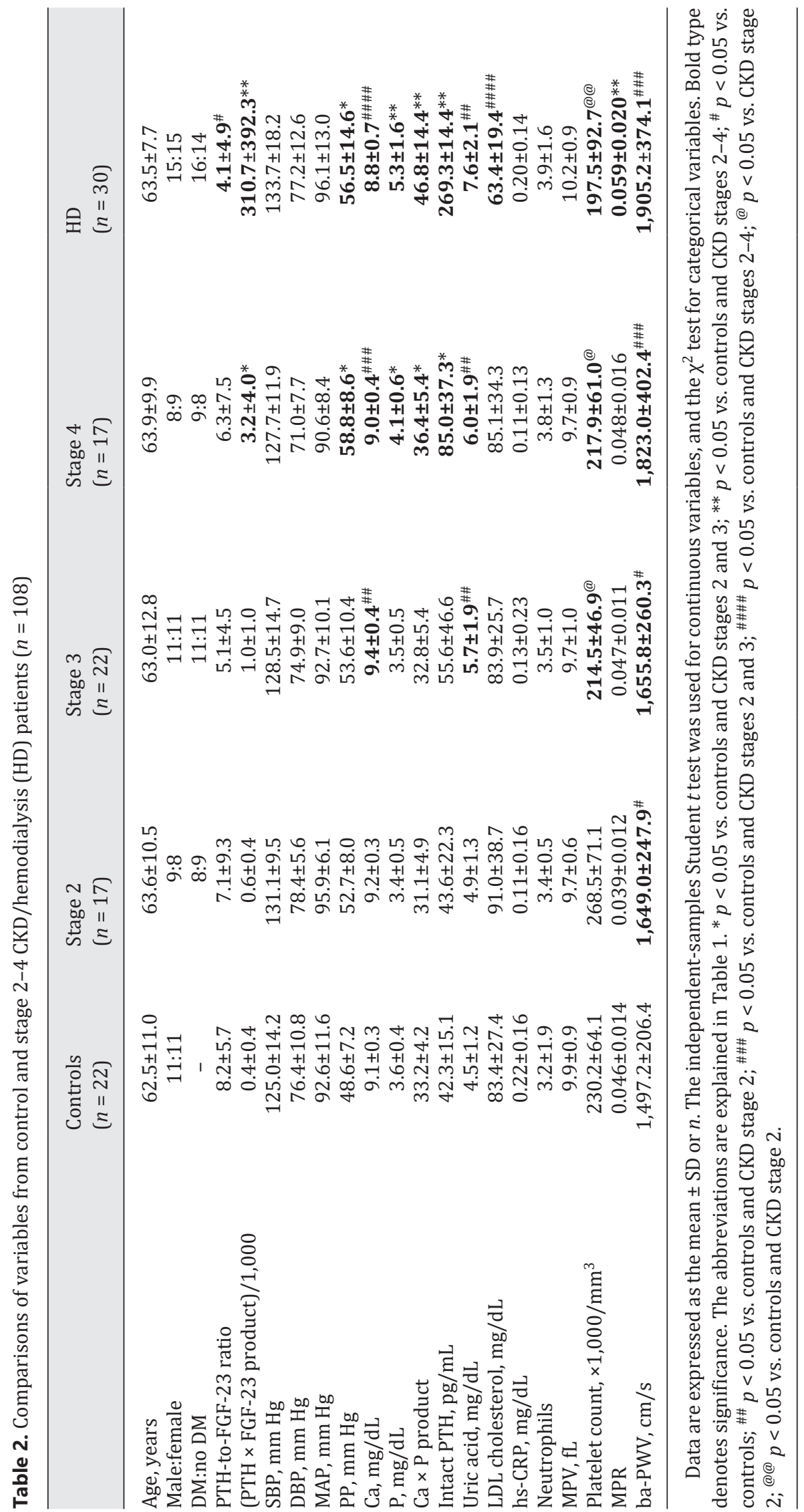


Kidney

Blood Pressure

Research

Fig. 1. Comparison of $\log _{10}$ (FGF-23) according to the chronic kidney disease (CKD) stage: control $(n=$ $22)$, stage $2(n=17)$, stage 3 ( $n=$ $22)$, stage $4(n=17)$, and hemodialysis (HD) $(n=30)$. Data are presented as the mean \pm SEM. ${ }^{*} p<$ 0.05 vs. control, stage 2 ; ${ }^{* *} p<0.05$ vs. control, stage 2,3 ; *** $p<0.05$ vs. control, stage $2,3,4$.

Fig. 2. Comparison of angiogenin concentrations according to chronic kidney disease (CKD) stage: control $(n=22)$, stage 2 $(n=17)$, stage $3(n=22)$, stage 4 ( $n=17)$, and hemodialysis (HD) $(n=30)$. Data are presented as the mean \pm SEM. $* p<0.05$ vs. control; ** $p<0.05$ vs. control, stage 2 ; *** $p<0.05$ vs. control, stage 2,3 .

Fig. 3. Comparison of the elastase/neutrophil ratio according to chronic kidney disease (CKD) stage: control $(n=22)$, stage 2 $(n=17)$, stage $3(n=22)$, stage 4 $(n=17)$, and hemodialysis (HD) $(n=30)$. Data are presented as the mean \pm SEM. ${ }^{*} p<0.05$ vs. control, stage 2 .

\begin{tabular}{l|l}
\hline Kidney Blood Press Res 2019;44:1166-1178 \\
\hline DOI: 10.1159/000502526 & $\begin{array}{l}\text { (c) 2019 The Author(s). Published by S. Karger AG, Basel } \\
\text { www.karger.com/kbr }\end{array}$ \\
\hline
\end{tabular}
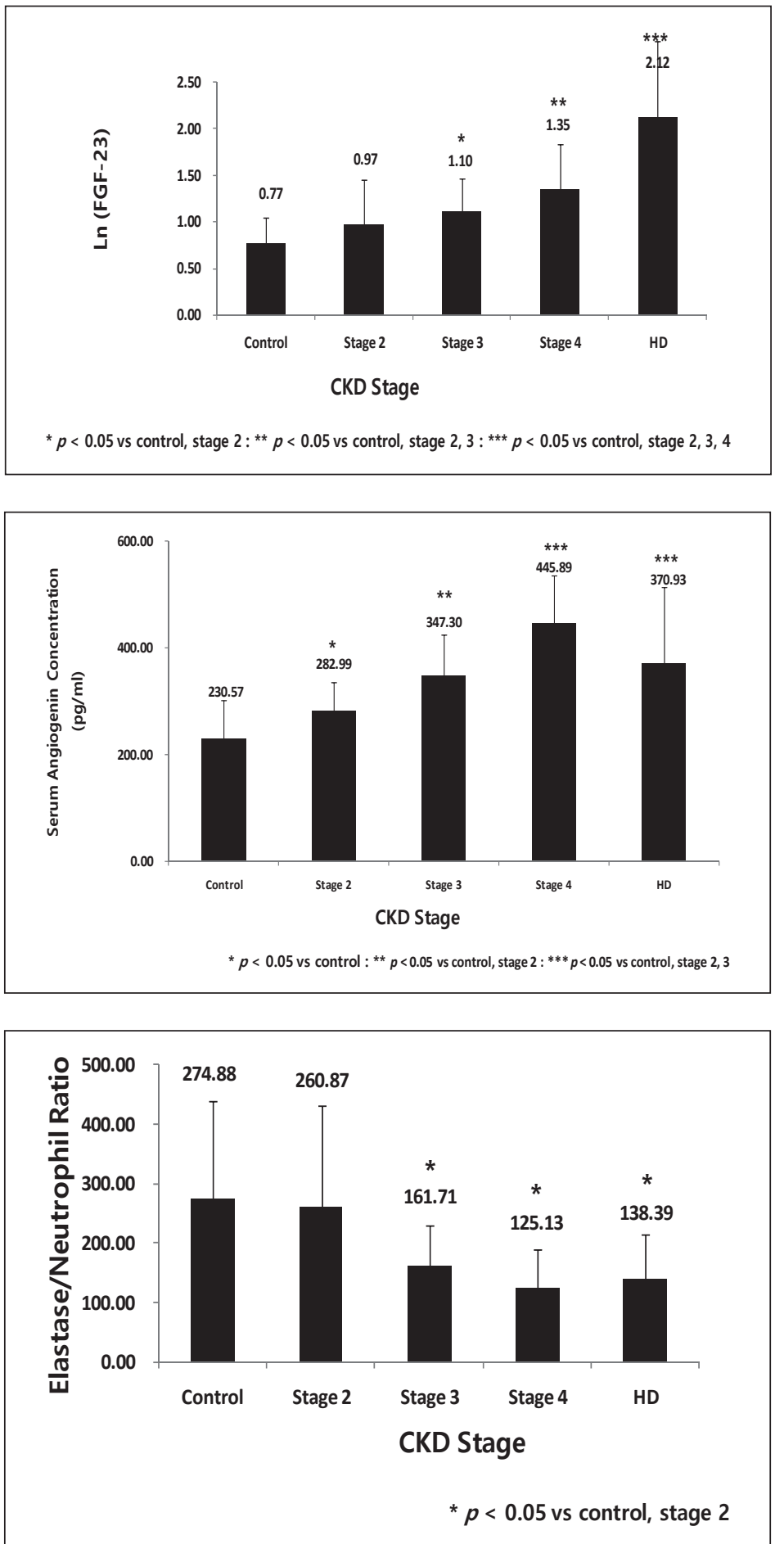

\section{Discussion}

This is the first study to describe circulating levels of FGF-23, elastase, lactoferrin, and angiogenin with respect to the various stages of CKD. Our results show that FGF-23 and angiogenin levels increased significantly with CKD progression. However, in the stage 3-4 CKD/HD patients, the elastase-to-neutrophil and lactoferrin-to-neutrophil ratios were found to be 
Kidney

Blood Pressure

Research

\begin{tabular}{l|l}
\hline Kidney Blood Press Res 2019;44:1166-1178 \\
\hline DOI: 10.1159/000502526 & $\begin{array}{l}\text { @ 2019 The Author(s). Published by S. Karger AG, Basel } \\
\text { www.karger.com/kbr }\end{array}$ \\
\hline
\end{tabular}

Choi et al.: FGF-23 in Arterial Stiffness

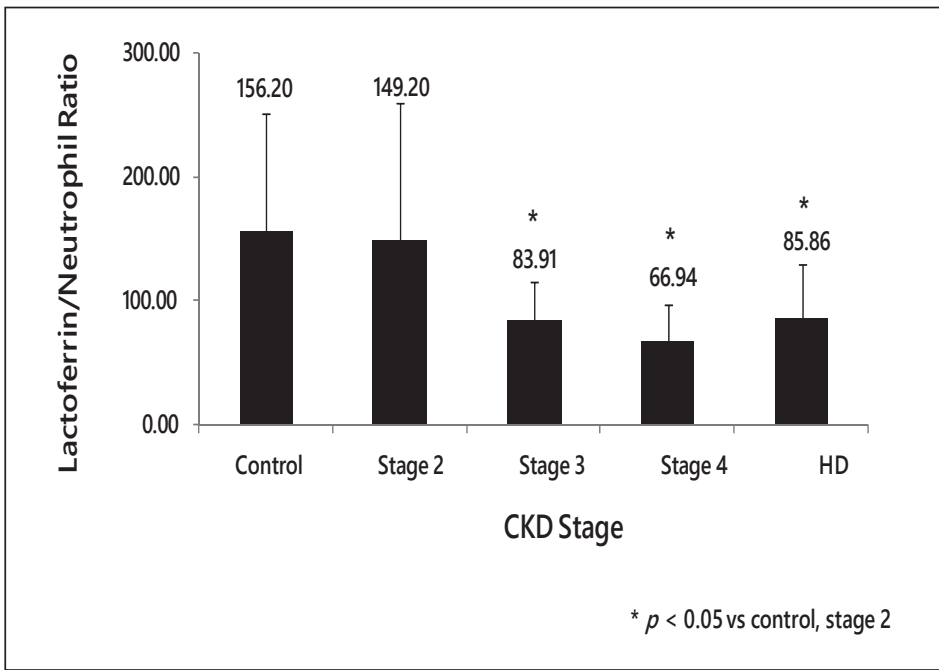

Table 3. Correlations of clinical and biochemical variables with ba-PWV in stage 2-4 CKD/hemodialysis patients $(n=86)$
Fig. 4. Comparison of the lactoferrin/neutrophil ratio according to chronic kidney disease (CKD) stage: control $(n=22)$, stage 2 ( $n=17)$, stage $3(n=22)$, stage 4 $(n=17)$, and hemodialysis (HD) $(n=30)$. Data are presented as the mean \pm SEM. $* p<0.05$ vs. control, stage 2 .

\begin{tabular}{lcl}
\hline Variables & $r$ & $p$ \\
\hline Age (years) & $\mathbf{0 . 4 8 7}$ & $\mathbf{0 . 0 0 1}^{*}$ \\
Sex (male:female) & -0.074 & 0.259 \\
Presence of DM & 0.172 & 0.066 \\
CKD stage & $\mathbf{0 . 4 3 9}$ & $\mathbf{0 . 0 0 1}$ \\
Elastase-to-neutrophil ratio & -0.038 & 0.369 \\
Lactoferrin-to-neutrophil ratio & 0.038 & 0.371 \\
Angiogenin (pg/mL) & -0.075 & 0.256 \\
log 1 (FGF-23) & $\mathbf{0 . 2 4 3}$ & $\mathbf{0 . 0 1 6}$ \\
Intact PTH (pg/mL) & $\mathbf{0 . 3 8 1}$ & $\mathbf{0 . 0 0 1}$ \\
(PTH × FGF-23 product)/1,000 & $\mathbf{0 . 2 0 0}$ & $\mathbf{0 . 0 4 0}$ \\
PTH-to-FGF-23 ratio & -0.178 & 0.060 \\
SBP (mm Hg) & $\mathbf{0 . 3 7 9}$ & $\mathbf{0 . 0 0 1}$ \\
DBP (mm Hg) & 0.134 & 0.121 \\
MAP (mm Hg) & $\mathbf{0 . 2 5 6}$ & $\mathbf{0 . 0 1 2}$ \\
PP (mm Hg) & $\mathbf{0 . 2 9 4}$ & $\mathbf{0 . 0 0 5}$ \\
Ca (mg/dL) & -0.150 & 0.095 \\
P (mg/dL) & $\mathbf{0 . 2 5 0}$ & $\mathbf{0 . 0 1 4}$ \\
Ca $\times$ P product & $\mathbf{0 . 2 2 2}$ & $\mathbf{0 . 0 2 5}$ \\
Uric acid (mg/dL) & 0.083 & 0.236 \\
LDL cholesterol (mg/dL) & -0.277 & 0.080 \\
MPR & 0.036 & 0.377 \\
\hline
\end{tabular}

Pearson's (parametric) correlations were used to test for associations of $\log _{10}$ (FGF-23) (due to skewed distribution of serum FGF-23 concentrations), elastase/lactoferrin-to-neutrophil ratio, MPR, angiogenin, and selected clinical, anthropometric and biochemical variables with ba-PWV. Bold type denotes significance. The abbreviations are explained in Table $1 .{ }^{*} p<0.05$.

significantly lower than in the controls and stage 2 CKD patients. Another important finding was that ba-PWV values showed a positive correlation with serum FGF-23 concentration; however, no correlation of serum elastase, lactoferrin, angiogenin, and MPR was found with ba-PWV values. Finally, FGF-23 was weakly associated with ba-PWV values. 


\section{Kidney \\ Blood Pressure \\ Research}

Table 4. Multivariate linear regression analysis with ba-PWV as the dependent variable in stage 2-4 CKD/hemodialysis patients $(n=86)$

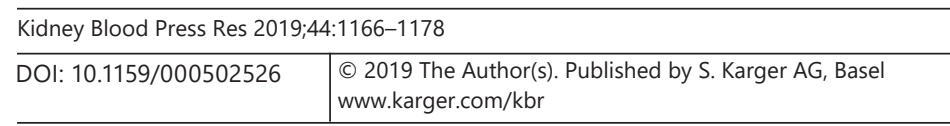

\begin{tabular}{llll}
\hline & \multicolumn{2}{l}{ ba-PWV } & \\
\cline { 2 - 4 } & $\beta$ & SE & $p$ \\
\hline Age & 16.405 & 3.801 & $\mathbf{0 . 0 0 1}^{*}$ \\
PP & 7.264 & 2.145 & $\mathbf{0 . 0 0 1}^{*}$ \\
MAP & 5.535 & 2.562 & $\mathbf{0 . 0 3 5}^{*}$ \\
PTH & 0.859 & 0.383 & $\mathbf{0 . 0 2 9}^{*}$ \\
$\log _{10}($ FGF-23) & 0.213 & 0.110 & $\mathbf{0 . 0 4 9}^{*}$ \\
\hline
\end{tabular}

Selected variables: age, sex, presence of DM, CKD stage, SBP, DBP, MAP, PP, uric acid, Ca, P, Ca $\times$ P product, PTH, LDL cholesterol, hs-CRP, $\log _{10}($ FGF-23), $($ PTH $\times$ FGF-23 product)/1,000, PTH-to-FGF-23 ratio, elastase-to-neutrophil ratio, lactoferrin-to-neutrophil ratio, angiogenin, and MPR. Bold type denotes significance. The abbreviations are explained in Table $1{ }^{*} p<0.05$.

FGF-23, a phosphatonin synthesized from osteocytes and osteoblasts, plays a key role in maintaining a normal P balance. In the advanced stages of CKD, serum FGF-23 levels are elevated to maintain serum $\mathrm{P}$ balance, and this increase is accompanied by an increase in serum PTH levels and a decrease in $1,25(\mathrm{OH})_{2}$ vitamin $\mathrm{D}_{3}$ levels. Gutierrez et al. [14] reported that an increase in serum FGF-23 levels was observed in patients with CKD in order to maintain a normal serum P balance as the eGFR values decrease. Similarly, a significant increase in serum FGF-23 levels was observed as the CKD stages advanced in our study. Ford et al. [15] reported that there was no relationship between increased serum FGF-23 levels and AS development as determined by PWV among predialysis CKD patients. Manghat et al. [16] suggested that there was no association between increased serum FGF-23 levels and AS development in predialysis CKD patients, and that the effect of increased FGF-23 in the predialysis period on the cardiovascular system was independent of its effects on the vessels.

In contrast to these studies, Mirza et al. [17] analyzed a community-based cohort of 967 persons aged 70 years and found that higher serum FGF-23 concentrations were associated with AS as measured by pulse wave analysis; this association was stronger in patients with impaired renal function ( $\mathrm{eGFR}<60 \mathrm{~mL} / \mathrm{min} / 1.73 \mathrm{~m}^{2}$ ). Coban et al. [4] showed that as the CKD stages advanced in ADPKD patients, AS development, determined by ba-PWV, increased compared to patients at earlier stages and to healthy individuals. Patients with high FGF-23 levels were found to have increased AS development compared to patients with low FGF-23 levels. The authors suggested that the difference in their results compared to other studies could be explained by the differences in CKD etiology and varying AS measurements.

Our results demonstrated that circulating FGF-23 concentrations were associated with AS. Although a recent experimental study found that protein levels of Klotho, the obligate coreceptor for FGF-23 in kidney and parathyroid tissues, were undetectable in arteries harvested from mice, and FGF-23 treatment did not affect arterial function in ex vivo studies of murine mesenteric arteries [18], our findings indicate the possibility of a direct trophic effect of FGF-23 on arteries. Therefore, whether FGF-23 plays a direct role in AS needs to be determined. Although our finding was statistically significant, the strength of association between FGF-23 and AS showed that the $\beta$ values were relatively lower than the values for age, PP, MAP, and PTH. Accordingly, our results suggest that there is no robust association between FGF-23 and AS, and the changes in AS in CKD patients may be caused by aberrant $\mathrm{Ca}$, $\mathrm{P}, \mathrm{PTH}$, or $1,25(\mathrm{OH})_{2}$ vitamin $\mathrm{D}_{3}$ homeostasis rather than by changes in FGF-23 levels alone.

Elastase and lactoferrin are contained within primary and secondary granules in neutrophils, respectively, and are frequently used as indirect markers of neutrophil activation in 


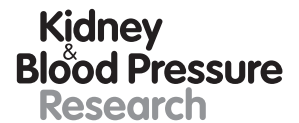

Kidney
Blood Pressure
Research \begin{tabular}{l|l}
\hline Kidney Blood Press Res 2019;44:1166-1178 \\
\hline DOI: 10.1159/000502526 & $\begin{array}{l}\text { @ 2019 The Author(s). Published by S. Karger AG, Basel } \\
\text { www.karger.com/kbr }\end{array}$ \\
\hline
\end{tabular}

Choi et al.: FGF-23 in Arterial Stiffness

vivo. Recently, many reports have used the ratios of elastase and of lactoferrin to the number of neutrophils, which are accurate markers of neutrophil activation. A previous report found that elastase was higher in CKD/HD patients, and that its value increased after HD sessions [7]. In particular, the long-term intradialytic contact of blood with large-surfaced artificial materials during HD may lead to leukocyte activation, with release of ROS and granule constituents, such as elastase [19]. Along these lines, it has been reported that higher serum levels of free elastase contribute to endothelial dysfunction [20]. These reports suggest that the neutrophil activation status may be linked with AS in patients with CKD/HD patients.

Unexpectedly, we found that the elastase-to-neutrophil and lactoferrin-to-neutrophil ratios were significantly lower in the stage 3-4 CKD/HD patients than in the controls and stage 2 CKD patients. In addition, the elastase- or lactoferrin-to-neutrophil ratio did not influence changes in AS. Previously, Costa et al. [19] demonstrated that the elastase- or lactoferrin-to-neutrophil ratio was not significantly different between their controls and HD patients. Although our study is the first to describe circulating levels of the elastase- and lactoferrin-to-neutrophil ratios with respect to the different stages of CKD, we cannot decide whether the circulating elastase- and lactoferrin-to-neutrophil ratios change as CKD progresses, nor confirm their biologic function on AS in CKD/end-stage renal disease patients, such that further study will be necessary.

Notably, angiogenin not only stimulates angiogenesis [21] but also exhibits anti-inflammatory and immunosuppressive activity [22]. Therefore, angiogenin not only stimulates the development of new vessels but also protects already existing vessels from the damaging impact of proinflammatory factors. In addition, it has also been shown that angiogenin binds to actin on the surface of endothelial cells, and that the angiogenin-actin complex stimulates tissue plasminogen activator to produce plasmin from plasminogen [23]. These observations indicate that angiogenin is an antithrombotic factor. Conversely, Burgmann et al. [10] found significantly increased serum concentrations of angiogenin in patients with stage IV PAOD compared to their control groups, patients with stage IIb PAOD, and patients with deep vein thrombosis. The authors concluded that serum angiogenin concentrations are increased in patients with stage IV PAOD and may be used as markers of disease severity.

Until now, there have been no data on serum levels of angiogenin in CKD patients without cardiovascular disease, as presented in the current study. Therefore, bearing in mind all the aforementioned properties of angiogenin, our observations shed new light on the development of AS. Our results show that plasma angiogenin concentrations increased as CKD advanced, whereas there was no significant association between plasma angiogenin concentration and AS.

The role of platelets in increased vascular stiffness has been much less well defined, especially in CKD patients [12]. Activated platelets release a variety of substances that control vascular permeability, regulate vasoconstriction or vasodilatation, stimulate macrophage recruitment and infiltration, release ROS, and stimulate ROS generation within the vascular wall, leading to decreased vascular nitric oxide bioavailability [24]. MPV has previously been examined as a platelet activation marker. It is known that larger platelets contain more granules and are in general enzymatically and metabolically more active than smaller ones, and consequently exhibit more prothrombotic characteristics. Several previous studies have found that MPV is strongly associated with AS in normotensive ADPKD patients and the general population $[12,25]$. However, our results show that although the MPR in the HD patients was significantly higher than in the control-to-stage 2-4 CKD subjects, there was no association between MPR and AS.

Before concluding, we would like to mention several limitations that may have adversely affected our study's results. Firstly, the study was conducted at a single center and with a low number of patients. Secondly, since the study was conducted at a cross-sectional time, the 
long-term effects of increased serum FGF-23 levels on AS in CKD/HD patients could not be investigated. Thirdly, since the patients continued to take their medication during the study period, it was not possible to investigate the negative effects of factors such as hypertension that contribute to AS development. Fourthly, AS development was assessed using a ba-PWV instrument only; the patients were not evaluated using a carotid-femoral PWV instrument, which is known to have higher sensitivity and is regarded as the gold standard.

In conclusion, circulating FGF-23 and angiogenin concentrations were found to progressively increase as CKD advanced, whereas the neutrophil activation markers in the stage 3-4 CKD/HD patients were found to be significantly lower than in the controls and stage 2 CKD patients. FGF-23 was weakly associated with ba-PWV values in patients with CKD/HD without a history of cardiovascular disease.

\section{Acknowledgments}

This work was supported by Kyowa Hakko Kirin Korea Co., Ltd. The authors would like to extend their gratitude to Kyowa Hakko Kirin Korea Co., Ltd.

\section{Statement of Ethics}

The subjects (or their parents and guardians) gave their written informed consent. The study protocol was approved by the research institute's Committee on Human Research (MJH201601123021-HE001).

\section{Disclosure Statement}

All the authors declare no competing interests.

\section{Author Contributions}

H.-M.C. designed the study; Y.-E.K. and S.K. collected the data; D.-J.O. drafted the manuscript.

\section{References}

1 Antonelou MH, Georgatzakou HT, Tzounakas VL, Velentzas AD, Kokkalis AC, Kriebardis AG, et al. Blood modifications associated with end stage renal disease duration, progression and cardiovascular mortality: a 3-year follow-up pilot study. J Proteomics. 2014 Apr;101:88-101.

2 Gungor O, Unal HU, Guclu A, Gezer M, Eyileten T, Guzel FB, et al. IL-33 and ST2 levels in chronic kidney disease: associations with inflammation, vascular abnormalities, cardiovascular events, and survival. PLoS One. 2017 Jun;12(6):e0178939.

3 London GM, Guérin AP, Marchais SJ, Métivier F, Pannier B, Adda H. Arterial media calcification in end-stage renal disease: impact on all-cause and cardiovascular mortality. Nephrol Dial Transplant. 2003 Sep;18(9): 1731-40.

4 Coban M, Inci A, Yılmaz U, Asilturk E. The Association of Fibroblast Growth Factor 23 with Arterial Stiffness and Atherosclerosis in Patients with Autosomal Dominant Polycystic Kidney Disease. Kidney Blood Press Res. 2018;43(4):1160-73.

5 Llauradó G, Megia A, Cano A, Giménez-Palop 0, Simón I, González-Sastre M, et al. FGF-23/Vitamin D Axis in Type 1 Diabetes: The Potential Role of Mineral Metabolism in Arterial Stiffness. PLoS One. 2015 Oct; 10(10):e0140222. 
6 Mourad JJ, Girerd X, Boutouyrie P, Laurent S, Safar M, London G. Increased stiffness of radial artery wall material in end-stage renal disease. Hypertension. 1997 Dec;30(6):1425-30.

7 Carollo C, Lo Presti R, Caimi G. Leukocyte activation markers and oxidative status in chronic kidney disease. Minerva Urol Nefrol. 2013 Mar;65(1):69-76.

8 Polańska B, Augustyniak D, Makulska I, Niemczuk M, Zwolińska D, Jankowski A. Elastase, $\alpha 1$-proteinase inhibitor, and interleukin-8 in pre-dialyzed and hemodialyzed patients with chronic kidney disease. Pediatr Int. 2010 0ct;52(5):735-43.

9 Costa E, Rocha S, Rocha-Pereira P, Nascimento H, Castro E, Miranda V, et al. Neutrophil activation and resistance to recombinant human erythropoietin therapy in hemodialysis patients. Am J Nephrol. 2008;28(6): 935-40.

10 Burgmann H, Hollenstein U, Maca T, Zedwitz-Liebenstein K, Thalhammer F, Koppensteiner R, et al. Increased serum laminin and angiogenin concentrations in patients with peripheral arterial occlusive disease. J Clin Pathol. 1996 Jun;49(6):508-10.

11 Kaya MG, Yarlioglues M, Gunebakmaz O, Gunturk E, Inanc T, Dogan A, et al. Platelet activation and inflammatory response in patients with non-dipper hypertension. Atherosclerosis. 2010 Mar;209(1):278-82.

12 Orscelik O, Kocyigit I, Akpek M, Dogdu O, Kaya C, Unal A, et al. Mean platelet volume and its relation with arterial stiffness in patients with normotensive polycystic kidney disease. J Investig Med. 2013 Mar;61(3): 597-603.

13 Yokoyama H, Shoji T, Kimoto E, Shinohara K, Tanaka S, Koyama H, et al. Pulse wave velocity in lower-limb arteries among diabetic patients with peripheral arterial disease. J Atheroscler Thromb. 2003;10(4):253-8.

14 Gutierrez O, Isakova T, Rhee E, Shah A, Holmes J, Collerone G, et al. Fibroblast growth factor-23 mitigates hyperphosphatemia but accentuates calcitriol deficiency in chronic kidney disease. J Am Soc Nephrol. 2005 Jul;16(7):2205-15.

15 Ford ML, Smith ER, Tomlinson LA, Chatterjee PK, Rajkumar C, Holt SG. FGF-23 and osteoprotegerin are independently associated with myocardial damage in chronic kidney disease stages 3 and 4 . Another link between chronic kidney disease-mineral bone disorder and the heart. Nephrol Dial Transplant. 2012 Feb;27(2):72733.

16 Manghat P, Souleimanova I, Cheung J, Wierzbicki AS, Harrington DJ, Shearer MJ, et al. Association of bone turnover markers and arterial stiffness in pre-dialysis chronic kidney disease (CKD). Bone. 2011 May;48(5): 1127-32.

17 Mirza MA, Larsson A, Lind L, Larsson TE. Circulating fibroblast growth factor-23 is associated with vascular dysfunction in the community. Atherosclerosis. 2009 Aug;205(2):385-90.

18 Lindberg K, Olauson H, Amin R, Ponnusamy A, Goetz R, Taylor RF, et al. Arterial klotho expression and FGF23 effects on vascular calcification and function. PLoS One. 2013;8(4):e60658.

19 Costa E, Rocha S, Rocha-Pereira P, Castro E, Reis F, Teixeira F, et al. Cross-talk between inflammation, coagulation/fibrinolysis and vascular access in hemodialysis patients. J Vasc Access. 2008 Oct-Dec;9(4):248-53.

20 Khatib-Massalha E, Michelis R, Trabelcy B, Gerchman Y, Kristal B, Ariel A, et al. Free circulating active elastase contributes to chronic inflammation in patients on hemodialysis. Am J Physiol Renal Physiol. 2018 Feb; 314(2):F203-9.

21 Marek N, Raczyńska K, Siebert J, Myśliwiec M, Zorena K, Myśliwska J, et al. Decreased angiogenin concentration in vitreous and serum in proliferative diabetic retinopathy. Microvasc Res. 2011 Jul;82(1):1-5.

22 Szcześniak P, Michalak Ł, Durasik A, Orszulak-Michalak D. Angiogenin and new markers of multivessel coronary arterial disease. Biofarm. 2009;65:83-7.

23 Tello-Montoliu A, Patel JV, Lip GY. Angiogenin: a review of the pathophysiology and potential clinical applications. J Thromb Haemost. 2006 Sep;4(9):1864-74.

24 Nording HM, Seizer P, Langer HF. Platelets in inflammation and atherogenesis. Front Immunol. 2015 Mar;6: 98.

25 Panova-Noeva M, Arnold N, Hermanns MI, Prochaska JH, Schulz A, Spronk HM, et al. Mean Platelet Volume and Arterial Stiffness - Clinical Relationship and Common Genetic Variability. Sci Rep. 2017 Jan;7(1):40229. 